\title{
Les pâturages de la région de Fianarantsoa (Madagascar)
}

\author{
par A. GILLARD
}

L'utilisation rationnelle des pâturages est conditionnée par leur étude botanique qui, en déterminant la nature des peuplements qui les constituent, permet d'aborder le problème visant à leur amélioration, et demeure, en fait, le principe élémentaire utile à l'établissement de tout programme valable se proposant de développer les grandes espèces domestiques.

Au cours d'un séjour de trois ans à Fianarantsoa, nous nous sommes attachés à rassembler la documentation pouvant nous permettre de dresser une carte des pâturages de la Province.

Notons qu'il s'agit d'une représentation schématique ayant pour but de les classer par grandes zones, définies suivant leurs dominantes fourragères propres.

Les lignes continues adoptées pour leur délimitation enlèvent forcément de la précision au document, mais nos moyens d'investigation étaient insuffisants pour réaliser d'emblée un travail plus complet.

C'est pourquoi, également, nous avons retenu l'échelle au $1 / 1.000 .000$ e pour la figuration de cette carte qui, bien que comportant d'inévitables lacunes, peut constituer néanmoins une base de départ pour des recherches ultérieures plus complètes.

N'apparaît-il pas dès maintenant souhaitable, en effet, après une étude statique plus minutieuse de nos prairies, de rechercher les relations pouvant exister entre les facteurs écologiques et les espèces rencontrées, d'en poursuivre systématiquement $l$ 'analyse afin de connaitre leur valeur nutritive aux divers stades de leur développement, d'ahorder enfin le problème plus délicat du phytodynamisme en relation avec la fauche, la fumure et les feux de brousse?

Ajoutons, en ce qui concerne ce dernier point, que la poursuite des essais relatifs à l'amélioration des pâturages à Aristida, mis en place à la ferme de l'Iboaka au début de 1951, doit nous aider à dégager dans quelques années d'intéressantes conclusions dans ce domaine.

Les espèces citées dans la présente note ont été déterminées par M. Bosser, agrostologue à l'Institut de la Rechercho Scientifique à Tananarive, auquel nous adressons nos plus vifs remerciements.

\author{
** \\ Les pâturages de la Province peuvent être classés \\ en cinq grands types qui sont, en allant de l'ouest \\ à l'est : \\ - Les pâturages à Danga et à Vero: \\ - A Kifafa et Trichopteryx; \\ - A Danga, Vero, Kifafa et Trichopteryx; \\ - A Ahipisaka et Tenina; \\ - A Ahipisaka, Tenina, Kifafa et Vero.
}

$\mathrm{Du}$ point de vue écologique, l'insuffisance de renseignements pédologiques nous a conduit à ne considérer, parmi les facteurs du milieu déterminants des patturages naturels, que les deux composants essentiels du climat : la pluviométrie et la température.

L'indice d'aridité de Martonne, calculé en fonction de la hauteur des précipitations et de la température, devait retenir notre attention en raison de l'analogie rencontrée entre la direction générale des courbes d'équiaridité et les divers types de pâturages identifiés.

Nous croyons également opportun, au début de cette note, de mentionner les difficultés de chiffrer avec précision le degré d'appétence des especes domestiques pour une plante, en raison de la compénétration des nombreux facteurs qui le déterminent.

Il varie, en effet, avec l'espèce, la race, l'individu, sa faim au moment consideré (charge des pâturages), le mode de distribution de la plante, la qualité des ressources fourragères de l'endroit, autant d'éléments qui conduisent bien souvent l'expérimentateur à des observations contradictoires.

C'est ainsi que le Trichopteryx stipoïdes, peu apprécié des zébus dans la zone à Danga, l'est beaucoup plus dans celle de Kifafa; que les raquettes inermes, recherchees du bétail dans le sud de l'île, sont négligées par lui dans la Province de Fianarantsoa; que dans un pâturage surchargé, Kifafa et Horompotsy sont consommés alors qu'ils sont délaissés dans la parcelle voisine disposant d'un petit nombre d'animaux et où, concurremment avec ces espèces, crôt en abondance le Vilona; qu'enfin l'Horompotsy, refusé par les animaux au pâturage 


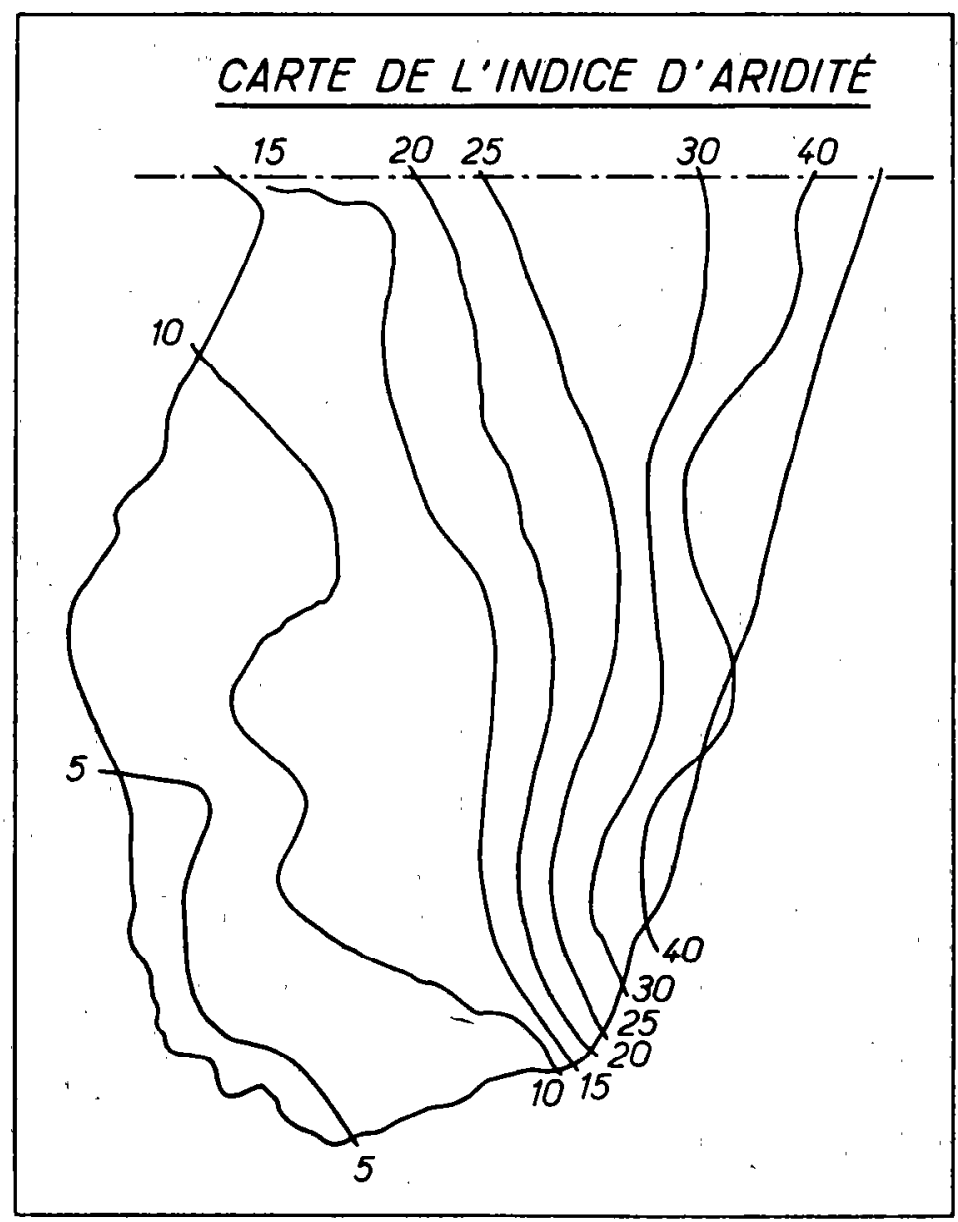

$$
\begin{aligned}
& A=\frac{P}{T+10} \\
& A=\text { Indice d'aridité de Martonne. } \\
& \mathrm{P}=\text { Pluie évaluée en millimètres. } \\
& \mathrm{T}=\text { Température en degrés centigrades. }
\end{aligned}
$$

SIGNIFICATION ÉCOLOGIQUE DE L'ÉCHELLE DES INDICES D'ARIDITÉ

$$
\begin{aligned}
A<5 & =\text { déserts, } \\
5<A<10 & =\text { steppes sèches, seule la culture irriguée est possible, } \\
10<A<20 & =\text { prairies, possibilité de dry farming, } \\
30<A & =\text { bonnes conditions d'existence pour la forêt, } \\
40<A & =\text { règne exclusif de la forêt. }
\end{aligned}
$$


où ils trouvent de meilleures espèces, est parfaitement consommé le soir, à l'étable, par ces mêmes animaux, lorsqu'on le leur distribue haché, en mélange avec de l'herbe Napier (Pennisetum purpureum).

Nous citons ces quelques exemples que nous pourrions multiplier à I'infini, afin de bien souligner la complexité du problème.

\section{PATURAGES A DANGa ET a VERO}

Cette région est caractérisée par un indice pluviométrique faible, une longue saison sèche (six à sept mois écologiquement secs), et une courte période de grande pluviosité (trois à quatre mois). En raison de l'altitude, on observe une saison fraîche, de mai à août, avec une amplitude thermique annuelle très forte (voir Tableau II, page 224).

Les pâturages à Danga et à Vero occupent, à l'ouest de la province, une bande longitudinale de 50 à $100 \mathrm{~km}$ de large au nord, $150 \mathrm{~km}$ au sud, dans une région où l'altitude moyenne oscille entre 700 et $1.100 \mathrm{~m}$ et qui correspond assez sensiblement à un indice d'aridité $\leq 20$ (consulter la carte de l'indice d'aridité ci-contre). Rappelons à ce sujet que $\mathrm{E}$. de Martonne a défini l'indice d'aridité par la formule :

$$
A=\frac{P}{T+10}(1)
$$

On établit le nombre $A \mathrm{l}$ corresporıdant aux moyennes annuelles de $P$ et de $T$, puis le nombre $A 2$ calculé à partir des valeurs de $\mathrm{P}$ ei de $\mathrm{T}$ du mois le plus sec. L'indice définitif $A$ est obtenu par la moyenne arithmétique des nombres $\mathrm{Al}$ et $\mathrm{A} 2$.

Toute cette région repose sur le massif cristallin des Hauts-Plateaux, au relief assez accusé avec, dans sa partie sud, la table de l'Horombe.

Si l'on s'en rapporte aux travaux de Perrier de la Bathie sur la flore agrostologique malgache (2) dans l'ouest, à la forêt primitive aurait succédé tour à tour :

\section{L'Hyparrhenia cymbaria (Stapf);}

$2^{\circ}$ L'Hyparrhenia rufa (Stapf), le Bothriochloa glabra ( $A$. Camus), l'imperata cylindrica (L.) et l'Heteropogon contortus (P. B.) sur les surfaces horizontales ou en pentes douces;

L'Imperata cylindrica et le Bothriochloa glabra en stations fraîches et riches;

(1) L'indice d'aridité à Madagascar par Pierre Duvergé (publication du Service Météorologique de Madagascar, septembre 1949).

(2) Perrier de la Bathie. - Les prairies de Madagascar. $3^{\circ}$ Enfin, l'Aristida sur les argiles latéritiques les plus pauvres.

Dans cette partie de la province de Fianarantsoa, nous rencontrons deux espèces essentielles :

Le Vero ( $H$. rufa, Stapf) et le Danga (Heteropogon contortus, P. B.), qui, tour à tour, dominent.

Cependant, si l'H. rufa s'associe volontiers au Danga sur les pentes et sommets des nombreuses collines des Hauts-Plateaux, nous rencontrons également comme espèces accessoires :

L'H. cymbaria (Stapf) et 1'1. cylindrica (L.) dans les bas-fonds humides et fertiles;

L'H. ruprechtii (F.) et l'H. lecomtei. (Stapf), tous les deux abondants dans le district d'Ambatofinandtahana:

Le Trachypogon polymorphus (Hackel), fréquent sur le plateau de 1'Horombe et assez bien appété des bovins lorsqu'il est jeune;

Le Schizachyrium ambalavense ( $A$. Camus), en peuplements denses dans le district d'Ambatofinandrahana, entre Amborompotsy et Ambatomainty, réputé médiocre fourrage;

Le $B$. glabra ( $A$. Camus), grégaire, rencontré surtout dans les endroits frais et passant pour donner un bon fourrage:

Le Trichopteryx stipoïdes (Hackel), peu abondant dans ce type de pâturages où il végète sous forme de touffes isolees:

Enfin l'Aristida dans les régions les plus dégradées.

Parmi ces espèces, le Danga, ou Ahidambo, est la plus estimée des éleveurs autochtones. Doit-on voir dans cette croyance une des raisons qui ont déterminé les Bara, peuple de pasteurs, à " suivre le Danga " au cours de leurs migrations vers le nord? Les Vero ont la réputation d'être excellents quand ils sont jeunes, mais après la fructification de ne donner qu'une herbe grossière, aux chaumes silicifiés, durs, et peu aimés du bétail.

Malgré leur caractère très accessoire, citons pour les avoir rencontrés :

L'Eragrostis chapelieri (Kunth) et le Chrysopogon montanus (Stapf), en petites touffes isolees et rares.

Les analyses effectuées par M. Philibert ( $H_{\text {. }}$, Chef du Laboratoire de Chimie et de Recherche des Fraudes à Tananarive, ont révélé, pour certaines espèces les compositions suivantes (voir Tableau III, page 225).

Autour des villages et sur les anciens emplacements de parcs à bœufs, le Cynodon dactylon (Pers.), especes nitratophile par excellence, anthropophile, résistante à la sécheresse et donnant un excellent fourrage;

Le Pennisetum polystachium (Sch.) et le Rhynchelytrum repens (Hubb.), sur le bord des chemins, à la lisière des cultures et sur les terres en friche; 
fournissent un excellent fourrage, la dernière espèce notamment, sous forme de foin;

Le Tricholaena monachne (Stapg et Hubbard), recherche de préférence les endroits frais;

Le Rottboellia exaltata (L.), plante annuelle végétant dans les terrains de cultures ou en friche, en station fraîche ou humide, et considéré par les Bara comme un bon fourrage lorsqu'il est jeune. Serait même consommé à l'état sec.

Parmi les rudérales appartenant au même groupe :

L'Eleusine indica (Gaertn) et le Sporobolus indicus (L.), peu fréquentes et sans grand intérêt dans l'alimentation du bétail.

L'Eragrostis cilianensis (Lut.), le Chloris madagascariensis (Steudel) et le Digitaria adscendens (Henr.) assez abondants, parfois, sur le bord des routes.

Dans les endroits humides, les bas-fonds, sur le bord des rivière's et cours d'eau :

L'Imperata cylindrica (L.), espèce pyrophile, ubiquiste, très envahissante, significative d'un sol peu dégradé et dont les jeunes pousses sont bien consommées par les bovins;

Le Phragmites mauritianus (Kunth), sorte de roseau pouvant atteindre $2,5 \mathrm{~m}$ à $3 \mathrm{~m}$ de haut, parfois abondant et dont les jeunes feuilles sont recherchées des bovins pendant la saison sèche;

L'Echinochloa pyramidalis (Hitch et Chase), vivace, hygrophile, abondant dans le marais d'thosy, sur les bords des rivières Manantanana, Zomandao, Sahambano, etc. Très recherché des zébus durant la période sèche (août à septembre);

Le Panicum maximum (Jacq.), très estimé du bétail, crô̂t en station humide ou fraîche, sur les alluvions et limons, les bords des chemins et cours d'eau;

Le Sorghum verticilliflorum (Stapf), sur alluvions riches. Il a la réputation, en pays Bara, de faire crever les bœufs lorsqu'il est consommé jeune, confirmant ainsi les observations de Coliman qui, analysant divers sorghos du Queensland, en 1934, trouvait la plus forte teneur en $\mathrm{H}$. C. N. dans 'S. halapense et $S$. verticilliflorum. En ce qui nous concerne, pendant quatre jours consécutifs nous avons soumis un lapin au régime exclusif de jeunes pousses de S. verticilliflorum, sans résultat appréciable. Il convient à la vérité de dire que durant ce laps de temps, l'animal n'en avait consommé qu'une très petite quantité, préférant s'abstenir de toute nourriture.

L'on sait que les végétaux contenant un glucoside cyanogénétique sont très nombreux. Il y aurait là, semble-t-il, d'intéressantes recherches à poursuivre à Madagascar.

Ajoutons également la présence de nombreuses cypéracées, quelques ombellifères (hydrocotyles), acanthacées, commélinacées, labiées, cnothéracées, composées hydrophiles, plus ou moins bien acceptées du bétail et constituant, avec d'autres graminées : Panicum, Digitaria, etc., le fond de la flore des pâturages de saison sèche.

Au nombre des légumineuses identifiées:

Le Cassia mimosoïdes (L.), le Leptodesmia congesta (Benth), des Eriosema procumbens (Benthl), cajanoïdes (Guill. el Perr.), Indigofera tinctoria, richardiana, généralement délaissés ou peu appréciés des zébus. Signalons également l'existence d'un petit arbuște grêle, le Sesbania pubescens, fréquemment rencontré dans les endroits frais, le lit des rivières, et dont le feuillage nous a paru bien appété des zébus.

\section{PATURAGES A KIFAFA ET TRICHOPTERYX}

Région caractérisée par un indice pluviométrique moyen ou élevé, de fortes pluies de novembre à mars, une saison sèche de durée moyenne, tempérée par des bruines et des précipitations occultes importantes - brouillards, rosées - et dont le relief détermine un climat d'altitude comportant une longue saison fraîche, d'avril à octobre, et une forte amplitude thermique.

Comprise approximativement entre les indices d'aridité 25-30 au nord, 23-30 au sud, sans grand intérêt pour l'élevage, elle occupe néanmoins des superficies considérables correspondant aux terrains les plus dégradés.

Sa limite $E$ se superpose, pratiquement, à celle du Trichopteryx (voir Tableau IV, page 225).

En ce qui concerne l'évolution de la flore dans cette partie de l'île, Perrier de la Bathie admet qu'aux formations primitives du domaine central (forêt à mousses sur le versant oriental, sylve à lichens sur les. crêtes, broussailles éricoïdes sur les cimes), avaient succédé, sur le versant est, le "Savoka " à Phillippia, et sur les pentes plus sèches du versant ouest, la fougère avec Hyparrhenia cymbaria, l'H. rufa et l'Imperata; l'Aristida, enfin, comme terme final.

Dans ce type de pâturage et comme eșpèces principales :

L'A. similis (Steudel), croît șurtout dans la partie nord de Fianarantsoa.

Les $A$. rufescens (Baron) et multicaulis (Baron), au sud (voir composition du Tableau V, page 226);

Le T. stipoïdes (Hackel), vivace, pyrophyte, semble ici dans son habitat de prédilection. En association avec les espèces précédentes, on le rencontre en maints endroits sous forme de vastes peuplements où îl domine d'une façon indiscutable.

Du point de vue fourrager, les éleveurs l'estiment très supérieur à l'Aristida que les animaux délaissent dès qu'il a fructifié. 
Comme especes accessoires importantes :

L'Imperata cylindrica, d'autant plus abondant que l'on se rapproche de la forêt;

Dans les districts d'Ambositra, de Fandriana et d'Ambatofinandrahana :

L'Elionurus tristis (Hackel);

Le Schizachyrium ambalavense (A. Camus);

Le $S$. semiherbe (Nees);

Espèces généralement confondues sous le même vocable d' "Ahitsorohitra " (herbe à alouettes), à souches vivaces, à rejets grêles et courts, souvent rougeâtres; ont la réputation d'être amères « mangidy », et seraient délaissées par le bétail aux dires des Betsileo.

Les Bara et les Antaisaka, qui connaissent bien le $S$. ambalavense, ne partagent pas cet avis puisqu'ils le nomment "Ahiborisoa " signifiant " herbe bonne et courte ".

Le T. polymorphus, abondant dans les districts d'Ambositra et d'Ambatofinandrahana, parfois en peuplements presque purs, le plus souvent en association avec l'Aristida similis et le Trichopteryx stipoìdes.

Certains Betsileo l'appellent « Ahibotolo » qui peut se traduire par " herbe grossière ». Les jeunes pousses sont bien consommées.

L'Andropogon trichozygus (Baker), abondant dans le canton de Sahave (District d'Ambohimahasoa) et le poste d'Ivohibe (District de Farafangana); il passe pour être une bonne espece fourragère.

Parmi les espèces accessoires secondaires ou rares :

L'Hyparrhenia rufa, en touffes isolées ou en plages plus importantes en station fraiche et riche;

L'H. lecomtei (Stapf), abondant dans certains pâturages d'Ambositra;

Le -Cymbopogon cymbarius, dans les endroits frais et riches, sur le bord des chemins:

L'Eulalia villosa (Nees), surtout fréquent vers l'est, près de la forêt. Certains propriétaires lui font la réputation d'être un bon fourrage alors que d'autres prétendent qu'il n'engraisse pas bien les boeufs:

L'Eragrostis brizoïdes ( $\mathrm{I}_{1}$ ), en station boisée ou récemment déboisée ; serait peu appété des bovins :

Le Sporobolus subulatus, surtout près de la forêt ;

L'Andropogon eucomus (Nees), en touffes isolées et rares, consommé par les bovins:

Le Melinis minutiflora (P. B.), réputé bon fourrage par les Bara; espèce vivace, à odeur accusée de mélasse, assez répandue dans la province, mais rencontrée le plus souvent sous forme de touffes isolées :

Le Schizachyrium brevifolium (Nees), le Craspedorhachis africana (Benth), l'Oxyrhachis gracillima
(Hubb.), et le Ctenium concinnum (Nees), rencontrés surtout dans les districts d'Ambositra, Ambatofinandrahana et Fandriana :

L'Alloterepsis semialata (Stapf), dans les pâturages des districts de Fandriana et Ambositra. Consommé par les bovins ;

Le Neyraudia madagascariensis (Kack.), vivace, reeherchant les endroits frais et riches;

L'Heteropogon contortus, en touffes isolées et rares:

Le Chrysopogon montanus, déjà cité ;

Le Sporobolus festivus (Hochwetter), exceptionnel.

Autour des villages, nous retrouvons les mêmes espèces que dans la zone précédente.

Dans les endroits humides, les bas-fonds périodiquement inondés, sur le bord des rivières et cours d'eau, dans les rizières.

Le Leersia hexandra (Sw.), une des meilleures espèces de la province; Mlle Camus (A.), dans son opuscule sur les graminées des prairies de Madagascar, a signalé sa résistance aux feux de brousse. En raison même de son habitat, il paraît peu vraisemblable qu'elle soit fréquemment soumise aux feux. Soulignons également son caractère ubiquiste puisqu'on la trouve dans tous les districts du territoire;

Le Cynodon dactylon, précédemment cité ;

Le Danicum glabrescens (Steudel), abondant dans les rizières ;

Le $P$. glanduliferum (Schum);

L.e Digitaria humbertii (A. Camus);

Le D. adscendens (Henr.);

Le D. sanguinalis (Stapf);

Le Brachiaria distachya (Stapf);

Le Sacciolepis interrupta (Stapf);

Le Poa annua (L.), de petite taille, produisant une herbe courte;

Le Cyrtococcum deltoildeum ( $\AA$. Camus);

L'Eriochloa acrotricha (Hackel);

sont toutes d'excellentes espèces fourragères, très recherchées du bétail.

Le $P$. maximum, fréquent sur les bords des routes et chemins dans les sols riches et frais;

Le Pennisetum pseudotriticoïdes ( $A$. Camus), espèce pyrophite, vivace, abondante autour et dans les rizieres abandonnées. Donne un fourrage ligneux, de mauvaise qualité (voir composition au Tableau $\mathrm{V}$, page 226).

A l'est, vers la forêt, dans les endroits ombragés :

Le Stenotaphrum dimidiatum (R.), très intéressante comme espèce fourragère;

L'Echinochloa colonum (Linck), sur, le bord des chemins, autour des habitations;

$L^{\prime} E$. stagnina (P. de B.), très bonne espèce fourragère, mais rare sur les plateaux;

Le Brachiaria mutica (Stapf), plante hygrophile, 
stolonifèr $\theta$, commune dans les cours d'eau et endroits humides, donnant un bon fourrage.

Le Commelina nudiflora (L.), à petites fleurs bleues:

Le C. Iyallii (H. Perrier), à fleurs jaunes, tous deux hygrophiles et appréciés des bovins.

Parmi les espèces rudérales, anthropophiles :

Le Cynodon dactylon;

L'Eragrostis chalcantha, le $P$. luridum (Hack) et le $D$. longiflora (Pers.), espèces rencontrées dans les terrains de cultures, près des villages, donnant un fourrage de qualité mais en quantité négligeable;

L'E. namaquensis (Nees), espèce assez rare dans la province;

Le Setaria pallidefusca (Stapf et Hubb.), annuel, croît sur le bord des routes, dans les terrains de cultures;

Le P. polystachyum, précédemment cité;

L'Eleusine indica;

L'E. curvula (Nees);

Le Sporobolus indicus (R. Br.), peu vivace, très répandu mais peu abondant; donne un mauvais fourrage;

Le Chloris pycnothryx (Trinius), peu fréquent;

Le Sida rhombifolia, de la famille des malvacées, à tiges ligneuses, mais dont les jeunes pousses sont bien consommées par les bovins;

Parmi les composées :

Le Bidens pilosa (Linne), abonde dans les terres cultivées; très apprécié des bovins et mieux encore des lapins;

Plusieurs espèces appartenant au genre Conyza, très répandues dans tous les terrains de cultures;

Le Galinsoga parviflora (Cav.);

L'Asystasia coromandeliana (Nees), de la famille des acanthacées;

Toutes consommées par les zébus.

Dans toute la zone à Aristida, les cultures étant beaucoup plus importantes qu'ailleurs, et les pâturages plus pauvres, ces espèces, ainsi que celles qui poussent dans les rizières, lc long des cours d'eau, jouent un rôle important dans l'alimentation des bovidés.

En ce qui concerne les légumineuses, aux espèces qui ont été citées pour la zone à Danga et à. Vero, il convient d'ajouter :

Le Vigna angivensis (Baker), consommé par les bovins ;

Des Tephrosia : retamoïdes (Sol), lyallii (Baker);

Des Desmodium : frutescens (Schindler), hirtum (Guill. et Pers.) et mauritianum (De Candolle), espèces semi-ligneuses, pyrophiles, et dont les jeunes pousses sont parfaitement acceptées du bétail. En ce qui concerne le $D$. frutescens, il nous a été donné de le contrôler, sur les bovins, équins, porcins et lapins;
L'Aeschynomene brevifolia (Poiret);

Le Zornia diphylla (Person); espèce consommée par les bovins, équins et lapins.

\section{PATURAGES A DANGA, VERO, KIFAFA ET TRICHOPTERYX}

Région infermédiaire entre les deux précédentes, où l'on rencontre, dominantes, toutes les espèces appartenant aux pâturages à Danga et à Vero avec, en plus, mais un petit nombre d'entre elles seulement et en franche minorié, celles appartenant à la zone à Kifafa et Trichopteryx (voir Tableau VI, page 226).

La limite Est de cette zone est celle au-delà de laquelle, pratiquement, le Danga.ne rencontre plus les conditions écologiques favorables à son développement. L'on en retrouve bien çà et là quelques touffes, voire en certains endroits, de perits peuplements, mais ils végètent mal et n'offrent guère qu'un intérêt botanique.

\section{PATURAGES A AHIPISAKA ET TENINA}

Région placée sous l'influence des alizés et caractérisée par un indice pluviométrique très élevé ou considérable, avec d'importantes pluies de relief déterminant une saison de grande pluviosité assez longue ou longue.

Saison fraîche de mai à septembre, avec une amplitude thermique moyenne (voir Tableau VII, page 227).

Cette zone se superpose assez exactement à la forêt, c'est pourquoi la carte forestière de la province, mise à notre dispostion par M. Vignal, Chef du Service Provincial des Eaux et Forêts, auquel nous sommes heureux, ici, d'exprimer nos vifs remerciements pour son amical concours; s'est révélée précieuse en la circonstance. Il va sans dire que les espèces fourragères appartenant à cette région, n'y sont pas uniformément réparties; on ne les rencontre guère que dans les endiroits déboisés, autour des villages, en lisières des massifs forestiers, le long des cours d'eau, routes, chemins et sentiers: C'est donc pour faciliter l'exécution de cette carte, que nous désirons schématique, que nous avons fait entièrement correspondre ce type de pâturages avec la forêt.

Deux espèces essentielles : l'Ahipisaka et le Tenina.

L' $\Lambda$ hipisaka groupe sous le mêne nom :

Le Stenotaphrum dimidiatum (R. Br.), l'Axoriopus compressus (P. B.) et le Paspalum conjugatum (Berg.), espèces ombrophiles, vivaces, stolonifères, recherchant les endroits frais et susceptibles d'un assez grand développement dans les sols riches. Considérées avec raison comme de bonnes espèces fourragères; il nous a été donné de voir, 


\section{GARTE SCEEMATIOUE DES PATURAGES DE EA PROVINCE DE FIANARANTSOA}

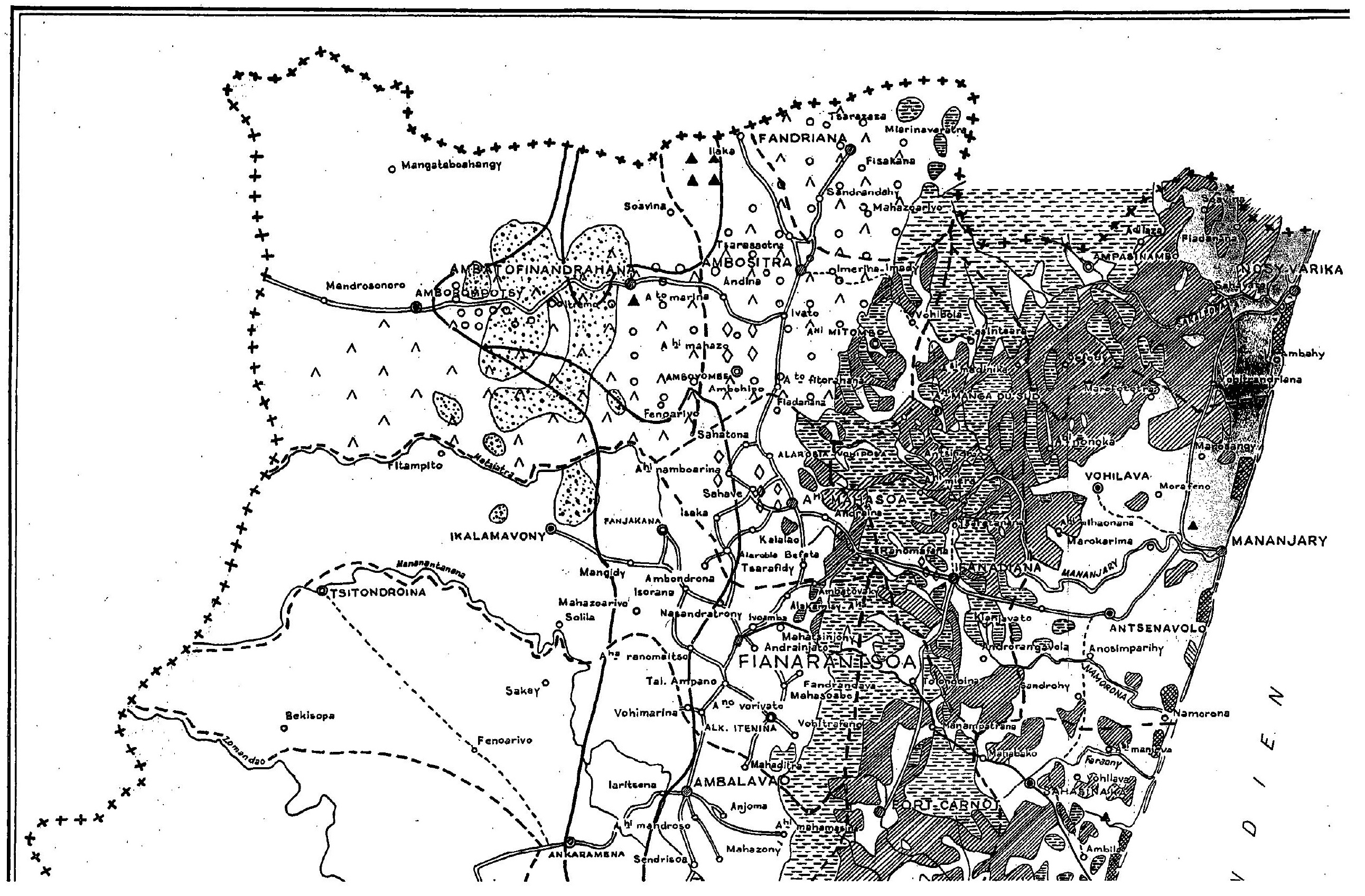




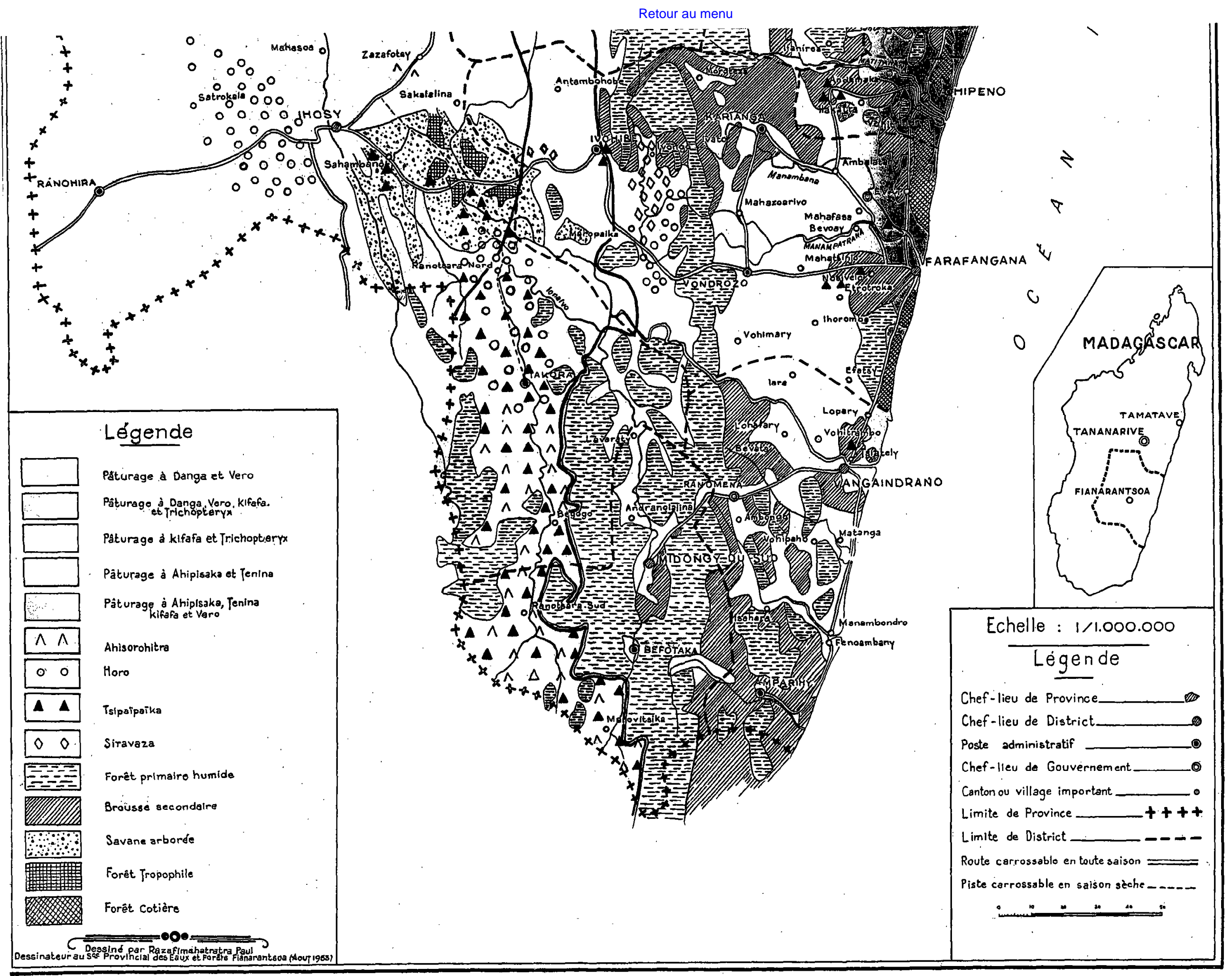


à l'île Maurice, d'excellentes laitières importées qui, durant six mois de l'année, consommaient exclusivement comme fourrage le $S$. dimidiatum, formant sous les filaos (Casuarina equisetifolia) du littoral, un tapis végétal épais et dense.

Le Tenina (Imperata cylindrica), pyrophile, grégaire, envahissant, dont les jeunes pousses sont bien consommées par les bovins et qui, d'après Eichinger contiendrait $5,8 \%$ de protéine pure (1).

Au nombre des espèces secondaires importantes:

Le Panicum maximum, l'Hyparrhenia rufa, l'H. cymbaria, l'Aristida similis, le Pennisetum polystachyum, précédemment cités :

Le P. paniculatum (L.) recherchant les sols riches; consommé par les bovins mais peu estimé, aux dires des éleveurs;

Le Neyraudia madagascariensis, assez fréquent; Une convolvulacée du genre Ipomea (patates), dont les bceufs et les lapins sont particulièrement friands ;

L'Urena lobata (L.) appartenant à la famille des malvacées et consommé par les zébus:

Le Sida rhombifolia, déjà cité.

Parmi les espèces secondaires moins importantes :

Le Commelina nudiflora,

Le f'loscopa glomerata (Hassk),

plantes hygrophiles, appréciées du bétail;

L'Andropogon eucomus, en touffes isolées et rares:

L'A. trichozygus, vivace, commun dans l'est du canton de Ranomafana (district d'Ifanadiana);

L'Hemarthria altissima (Staf et Hubb), espèce fourragère de sous-bois, recherchant les endroits frais (bord des rivieres).

Autour des villages :

Le Cynodon dactylon, l'Eleusine indica, le Sporobolus indicus, le Setaria pallidifusca, l'Eragrostis tenella, espèces anthropophiles, nitratophiles déjà citées:

Le S. chevalieri (Stapf), graminée vivace, à larges feuilles, consommée par les bovins:

Le $P$. commersonii (Lamk).

Sur les bords des cours d'eau :

Le Brachiaria mutica (Stapf) ou herbe de Para, vivace, stolonifère, très abondant dans le district d'Ifanadiana, fournissant un excellent fourrage;

Le Phragmites mauritianus, précédemment cité;

Le Coix lacryma jobi (L.), à graines grises, ovoides, de la grosseur d'un grain de maïs, et dont le feuillage est bien appété. Serait originaire de l'Asie tropicale.

(1) Graminées des prairies de Madagascar par A. Camus.
Enfin, deux cypéracées de grande taille, fréquentes dans les districts d'Ifanadiana et de FortCarnot, et que nous avons vu consommées par des zébus :

Le Cyperus latifolius (Poir.) et le Cyperus immensus (Clarke) dont les hampes florales sont également utilisées à la confection de chapeaux, nattes et vans.

Dans les terrains de cultures :

Le Leersia hexandra, plante aquatique, très abondante dans les rizières et, comme nous l'avons vu, excellente du point de vue fourrager;

L'Echinochloa colonum (Link), annuel, fréquent dans les caféières, endroits humides, avec :

Le $P$. glanduliferum;

Le Digitaria cæspitosa (Boiv.), ombrophile, formant un gazon fin, assez dense;

Le D. horizontalis;

Le $D$. adscendens;

Le Sacciolepis curvata (Chase);

donnant tous un excellent fourrage.

L'Eragrostis chalcantha et le Rhynchelytrum repens, déjà cités;

Le $P$. brevifolium (L.), espece annuelle, abondante dans les caféières et recherchée des zébus;

Le P. umbellatum (Trin.), moins abondant, vivace, à feuilles étroites, et formant un gazon ras; également bon fourrage;

L'Emilia citrina (D. C.) et l'Emilia humifusa (D. C.), composées abondantes dans les terrains de cultures (champs de manioc, plantations de caféiers), consommées par les bovins et très appréciées des lapins.

Mentionnons également parmi les cypéracées fréquemment rencontrées dans les rizières inondées : le Scirpus juncoildes (Roxb.), sorte de petit jonc, le Fuirena chlorocarpa (Ridl.), souvent consommées par les zébus.

Parmi les légumineuses, les Desmodium (frultescens-hirtum-mauritianum), appartiennent aux espèces les plus fréquentes.

\section{paturages a ahipisaka, tenina, KIFAFA ET VERO}

Région entièrement soumise à l'alizé austral, est caractérisée par un indice pluviométrique considérable, une saison seche nulle, des pluies réparties sur toute l'année déterminant une très longue saison de grande pluviosité marquée par un léger fléchissement en octobre.

Saison assez fraîche, de mai à octobre, avec une amplitude thermique moyenne ou forte (voir Tableau VIII, page 228).

D'après Perrier de la Bathie, cette région était autrefois couverte par la forêt orientale ayant des arbres de 20 à $30 \mathrm{~m}$. Soumise au régime du «tavy » par les autochtones, en vue de la culture du riz, 
cette magnifique forêt primitive fut peu à peu remplacée par le "Savoka ", végétation arbustive de 3 à $4 \mathrm{~m}$ de haut. Pour les mêmes raisons, le «Savoka " fut à son tour incendié faisant place à la prairie à Imperata, puis à Vero (Hyparrhenia rufa, H. cymbaria), avec, comme stade final, l'Aristida sinilis.

Nous distinguerons deux zones : l'une côtière, I'autre comprise entre celle-ci et la forêt orientale. Sur les sables de la régión cótière :

Le Stenotaphrum dimidiatum, l'Axonopus compressus, le Panicum umbellatum, les Commelina nudiflora et lyallii, dont il a été parlé plus haut;

Le C. madagascarica (Clarke), identifié également dans le district dithosy (zone à Danga et à Vero);

L'Ipomea pes caprae, convolvulacée fréquente sur le rivage; consommée par les bovins mais peut-être meilleure encore pour les lapins ;

Le Zoïsia tenuifolia (Wild.), espèce vivace croissant dans le sable et fournissant un pâturage court, mais apprécié;

Le Thouarea sarmentosia (Pers.), rampant, peu abondant; également consomme par les zebus;

Le Digitaria longiflora;

L'Ischaemum heterotrichum (Hackel), plus rare, réputé bon fourrage;

En station ombragée:

L'Hemarthria altissima (Stapf et Hubbard), rare;

Le Paśsiflora fotida (L.), de la famille des passifloracées et qui, selon les éleveurrs, serait bien consommé par les zébus.

Dans la région comprise entre la côte et la forêt orientale (jusqu'à 800 à $900 \mathrm{~m}$ d'alititude), nous reconnaissons comme espèces principales :

L'Aristida similis, couvrant de grands espaces sous forme de peuplements presque purs;

L'Hyparrhenia rufa, l'H. cymbaria, l'Imperata cylindrica, près de la forêt;

L'Hyparrhenia lecomtei, en peuplements denses, dans le canton dillakatra (district de Vohipeno).

Au nombre des espèces secondaires :

Le Panicum dregeanum (Nees), en association avec l'Aristidà similis depuis Nosy-Varika jusqu'à Vangaindrano:

L'Eragrostis chapelieri, I'Heteropogon contortus et le Bothriochloa glabra, dans le district de Farafangana surtout;

Près des villages, nous retrouvons à peu près la. même flore agrostologique que dans la zone précédente.

Le long des rivières, dans les régions inondées : Le Leersia hexandra;

L'Ischaemum rugosum (Sal.) qui, d'après Mille A. Camus, aurait probablement été introduit à Madagascar en 1914, avec le riz de Boïna ;

Le Brachiaria mutica, commun dans la partie nord:
L'Oriza madagascariensis ( $A$. Chevalier), rencontré sur les bords de la Sakaleona, près de NosyVarika; fournit un bon fourrage:

L'Echinochloa stagnina (P. de B.), très fréquent dans le district de Vohipeno (rizières, cours d'eau), où il est réputé excellent fourrage;

Le Coix lacryma-Jobi.

Dans les caféières :

Des Commélinacées, hydrocotyles, Panicum (maximum, brevifolium, umbellatum, glanduliferum), Paspalum (conjugatum, paniculatum), l'Echinochloa colonum; le Sacciolepis curvata, etc. En lisières des cultures : le Pennisetum polystachyum et le Rhynchelytrum irepeins.

Parmi les légumineuses, sur les sables du littoral :

Le Desmodium triflorum (De Candolle), ombrophile, formant un pâturage ras peu abondant, consommé par les zébus;

Des Tephrosia (T. purpurea-Pers.) (T. viciäldesRick.) qui seraient également consommés.

Entre la côte et la forêt :

Des Desmodium, especes semi-ligneuses;

L'Aeschynomene brevifolia (Poiret), le Cassia mimosoïdes.

Dans la prairie à aristida, le D. mauritianum (D. C.) est fréquent et nous avons pu observer sa remarquable résistance au feu. Les premières pluies font sortir de jeunes bourgeons que les zébus consomment volontiers.

\section{COMPOSITION DE LA FLORE DES PATURAGES}

Dans la province de Fianarantsoa, dont la superficie ne dépasse guère $100.000 \mathrm{~km}^{2}$, la flore agrostologiquo est largement dominée par les graminées, comparable en cela à celle de tous les pays tropicaux.

Bien qu'il soit hasardé de vouloir chiffrer leur importance, même relative, nous donnerons à titre indicatif les pourcentages suivants :

$$
\begin{aligned}
& \text { Aristida........... } 25 \text { à } 35 \% \\
& \text { Danga ............ } 20 \text { à } 30 \% \\
& \text { Vero.............. } 15 \text { à } 25 \% \\
& \text { Autres espèces...... } 20 \text { à } 30 \%
\end{aligned}
$$

Si l'on s'en tient aux travaux de Perrier de la Bathie sur l'evolution de la flore malgache au cours des siècles, qui considère la prairie à Aristida " comme le terme des dégradations successives auxquelles aboutissent sous l'action du feu - et nous ajouterons des cultures irrationnellement conduites les formations autochtones vierges i, nous pouvons d'ores et déjà conclure :

- Qu'une étendue considérable de la province de Fianarantsoa porte une végétation dont la dégradation a atteint son stade ultime: 
- Que ce faciès très dégradé est surtout rencontré dans la zone littorale et la partie centrale des Hauts-Plateaux.

Quelles sont les causes de ce processus accéléré de dégradation des sols?

Il apparait comme vraisemblable que si l'action du feu intervient efficacement dans l'épuisement des terres - et qu'en l'occurrence, il en demeure la raison première (destruction de la forêt primitive) - rien ne laisse à penser que cette région y ait été soumise avec plus d'intensité qu'ailleurs ou depuis des temps plus reculés.

Il semble plus admissible, au contraire, d'en rechercher l'origine parmi certains facteurs écologiques locaux propres à précipiter la dégradation des surfaces mises à nu par le feu (abondance des pluies) et peut-être aussi l'intervention malheureuse de l'homme par des cultures mal comprises (peuplement humain plus dense).

Dans toute cette partie, il ne s'agit pas simplement de conserver le sol, mais de le régénérer.

\section{RÉPARTITION COMPOSITION DES TROUPEAUX}

Si l'examen du tableau relatif au recensement de l'espèce bovine dans la province montre claire-

TABLEAU I. - RECENSEIMENT DE LTSPÈCE BOVINE

\begin{tabular}{|c|c|c|c|}
\hline DISTRICTS & $\begin{array}{l}\text { SUPERFICIES } \\
\left.\text { (en } \mathrm{km}^{2}\right)\end{array}$ & $\begin{array}{l}\text { TOTAL DES BOVINS } \\
\text { (recensement de 1953) }\end{array}$ & $\begin{array}{c}\text { NOMBRE } \\
\text { au } \\
\text { kilomètre carré }\end{array}$ \\
\hline 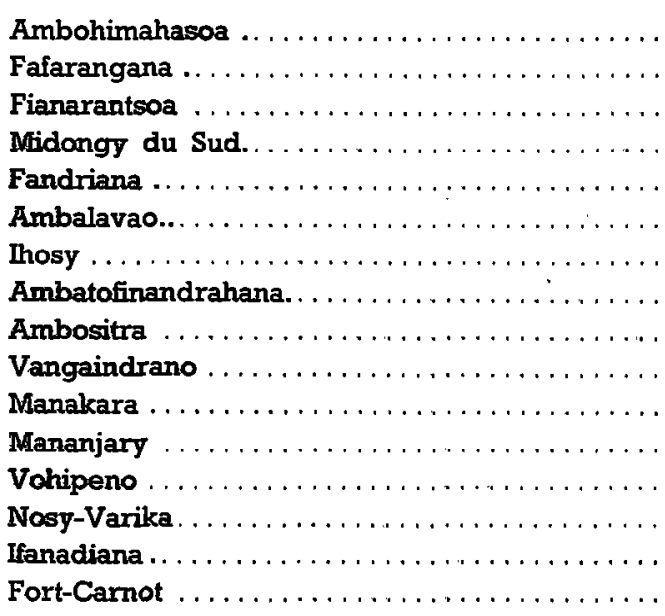 & $\begin{array}{r}1.800 \\
8.800 \\
8.900 \\
5.000 \\
2.400 \\
8.100 \\
20.000 \\
10.400 \\
7.500 \\
5.400 \\
3.000 \\
5.200 \\
2.000 \\
3.600 \\
5.100 \\
3.000\end{array}$ & $\begin{array}{r}43.047 \\
164.017 \\
118.659 \\
54.425 \\
26.006 \\
88.356 \\
156.691 \\
74.145 \\
51.320 \\
33.209 \\
16.295 \\
26.638 \\
9.963 \\
13.570 \\
1.289 \\
3.678\end{array}$ & $\begin{array}{r}23,9 \\
18,6 \\
13,3 \\
10,8 \\
10,8 \\
9,7 \\
7,8 \\
7,1 \\
6,8 \\
6,1 \\
5,4 \\
5,1 \\
4,9 \\
3,7 \\
1,4 \\
1,2\end{array}$ \\
\hline TOTAUX $\ldots \ldots \ldots \ldots \ldots \ldots$ & 101.200 & 887.338 & 8,7 \\
\hline
\end{tabular}

ment la pauvreté des districts forestiers en bétail, en raison même de l'occupation des terrains par la forêt, la qualité des pâturages, par contre, ne semble pas avoir d'incidence marquée sur l'importance des troupeaux. La zone à Kifafa, à population relativement dense, tout en étant de qualité indiscutablement médiocre, est également celle qui dispose du plus grand nombre de têtes au kilomètre carré. Par contre, il n'en est pas de même en ce qui concerne la composition des troupeaux.

En prenant au hasard, dans chacune des grandes régions types, une cinquantaine de milliers d'individus, nous relevons, par catégorie, les pourcentages suivants :

\section{I. - Zone à Danga et à Vero}

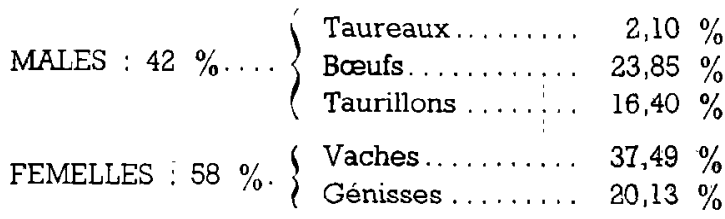




\section{II. - Zone à Kifafa et Trichopteryx}

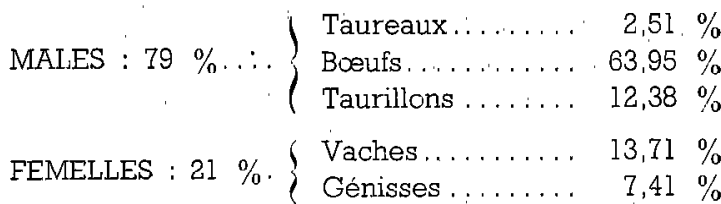

III. - Zone intermédiaire à Danga, Vero; Kifafa et Trichopteryx

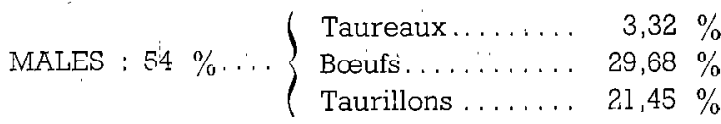

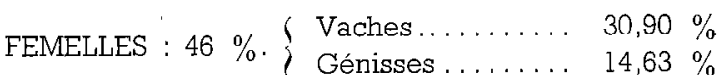

IV. - Zone à Ahipisạka et Tenina

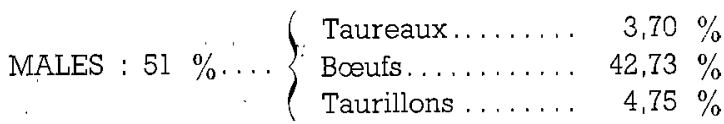

FEMELLES : $49 \%$. Vaches........... 40,67\%

V. - Zone à Ahipisaka, Tenina, Kifafa et Vero

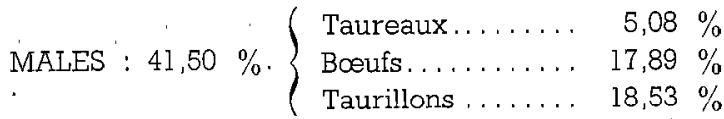

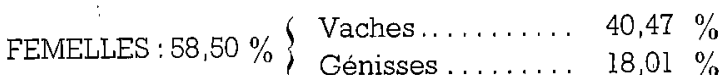

Dans la zone à Kifafa et Trichopteryx, pays essentiellement Betsileo, le boeuf "vositra 》 domine. Cette constitution particulière des troupeaux est souhaitable, si lion tient compte de la pauvreté des pâturages. I'apporl de paille de riz et de manioc, dans l'alimentation du bétail, loin d'être négligeable, est cependant insuffisante. C'est pourquoi, comme nous l'avons exprimé à maintes reprises, dans toute cette région, l'élevage doit nécessairement être intégré dans un système agricole.stable.

Aux cultures sèches itinérantes, doivent se substituer les cultures stabilisées. Pour y parvenir, il importe de recourii à la fumure organique.

La plante susceptible de fournir un fourrage de qualité en même temps qu'un bon engrais vert, bien adaptée aux conditions écologiques locales, doit être recherchée et partout répandue.

La zone à Danga et à Vero est la seule favorable à l'élevage. Une réglementation intelligente des feux de brousse (1) et la constitution de réserves

(1) Se reporter à l'étude très objective et très pertinente de notre confrère Metzger dont les remarquables observations sur ce sujet méritent l'attention et le concours efficient des Pouvoirs Publics. fourragères, simplement sous forme de foin, suffiraient pour transformer complètement l'élevage du zébu dans la province.

La région à Ahipisaka, Tenina, Kifafa et Vero, est, toutes choses restant égales par ailleurs, beaucoup moins intéressante, en raison des superficies considérables recouvertes par le Kifafa et de la surabondance des précipitations. Et nul doute que le simple bon sens - compte tenu des échecs permanents enregistrés dans cette zone - aurait dû, depuis bien longtemps déjà, orienter l'éleveur vers la constitution de troupeaux à prédominance de mâles.

Quant à la région à Ahipisaka et Tenina, dont l'importance actuelle est très limitée du point de vue élevage, ce dernier ne pourra s'avérer plus profitable sans la construction d'abris, le contrôle des naissances et la plantation d'especes fourragères, conditions réalisables ici, en raison de la moindre importance des troupeaux.

Fianarantsoa, le 25 octobre 1953.

\section{NOMS VERNACULAIRES DES ESPÈCES CITÉES}

Nous croyons utile d'appeler l'attention sur la fragilité des noms vernaculaires. Si, en effet, l'Imperata cylindrica (L.) et le Panicum maximum (Jacq.) sont unanimement désignés par Tenina, et Fataka; il ne s'agit là que d'exceptions.

Les dénominations locales sont généralement beaucoup moins simples. C'est ainsi, par exemple, quo le Danga ou Ahidambo qui correspond en Bara, Antaisaka, Hova, etc., à l'Heteropogon contortus (P. B.), est, pour certains Betsileo des districts d'Ambohimahasoa, d'Ambositra et d'Ambatofinandrahana, le Trichopteryx stipoïdes (Hackel) et pour d'autres, le Trachypogon polymorphus (Hackel).

Ailleurs, sous le même vocable, sont groupées des espèces très différentes. L'Ahipisaka peut aussi bien désigner pour les Tanala, Antaimoro, Antaisaka ou Antambahoaka, le Stenotaphrum dimidiatum (Br.), 1'Axonopus compressus (P. B.), voire le Paspalum conjugatum (Berg.).

Ces réserves formulées, nous indiquerons par ordre alphabétique les appellations locales les plus courantes des espèces dont il a été fait mention.

Alloteropsis semialata (Stapf). - Tongolonakanga (oignon de pintade) en Betsileo.

Andropogon eucomus (Nees), A. madagascariensis (Hackel). - Siravaza (sel gris) en Betsileo; Ahiborisoa (herbe courte et bonne) en Bara. Aristida similis (Steudel), Aristida rufescens (Baron), Aristida multicaulis (Baron). - Kifafa (balai) en Hova, Betsileo et Bara; Kamafa ou Komafa 
en Antaimoro, anala, Antaisaka, Antaifasy et Zafisoro; Famafa en Betsimisaraka.

Asystasia coromandeliana (Nees) (Acanthacée). Anamalemy (brede molle) en Betsimisaraka et Antambahoaka.

Axonopus compressus (P. B.). -- Ahipisaka.

Bidens pilosa (L.) (composée). - Tsipolitra en Hova, Trakavola (légumes d'argent) en Betsileo et Tanala; Traka en Bara et Antaisaka.

Brachiaria mutica (Stapf). - Zamana en Betsileo et Tanala.

Bothriochloa glabra (A. Camus). - Tsipaipaika, Ahisombilahy en Bara et Antaisaka du district de Midongy-du-Sud; Mafahitraomby en Antaimoro et Antaisaka du district de Farafangana.

Cassia mimosoïdes (L.). - Kisendrisendry en Bara. Coix lacryma-Jobi (L.). - Piko-Piko (perle qui pousse), Vakamaniry en Betsileo et Tanala.

Commelina nuditlora (L.), - Lyallii (H. Perrier) et madagascarica (Clarke). - Nifinakanga (dent de pintade) en Hova; Tsimativonoina en Betsileo et Tanala; Ahidomano (herbe qui nage) en Bara.

Craspedorhachis africana (Benth). - Ahitsorohitra en Betsileo.

Cymbopogon cymbarius (T. Thomson). - Verovato (vero de pierre) en Betsileo; Verombato en Bara.

Cynodon dactylon (Pers.). - Fandrotrarana en Hova; Kindresy en Betsileo et Bara; Ahipandotra en Betsimisaraka.

Cyperus immensus (Clarke) (cypéracée). - Vendrana en Tanala.

Cyperus latifolium (Poir.) (cypéracée). - Herana en Betsileo et Tanala.

Desmodium frutescens (Baker) (Légumineuse), Mandalodiaraikitra (on "passe et ça colle) en Hova et Betsileo; Sotromorona, en Tanala et Betsimisaraka; Tsipotiky en Bara.

Desmodium hirtum (Guill. et Pers.), - Singaka, Tsigatraka, Kianjoanjo en Tanala.

Desmodium mauritianum (De Candolle) (Légumineuse). - Tsilavondrivotra, Tsitolandrivotra (résistant au vent) en Tanala, Antaimoro, Antaisaka, Betsimisaraka, Bara.

Echinochloa pyramidalis (Hitch. et Chase). - Songolo, Tsongolo, en Bara.

Echinochloa stagnina (P. de B.). - Singarivary (image du riz) en Antaimoro; Kivarivary (même signification) en Tanala. En Afrique centrale, les graines seraient consommées en période de disette.

Eleusine indica (Gaertner). - Tsipipina ou Tsipihipihina en Hova et Betsileo; Ahitrombilahy (herbe de taureau) en Tanala, Antaisaka et Betsimisaraka.

Elionurus tristis (Hackel). - Ahitsorohitra en Betsileo.
Emilia citrina (D. C.) et E. humitusa (D. C.) (composées). - Tsiotsiona en Hova, Betsileo et Tanala; Tsiotsio en Antaimoro.

Eulalia villosa (Nees). - Gaibona, Mafaibaratra (la foudre amère) en Betsileo; Vodikongo en Bara. Galinsoga parviflora (Cav.) (composée). - Anapotsy (légume blanc) en Hova et Betsileo.

Heteropogon contortus (P. B.). - Danga en Hova; Ahidambo (herbe à sanglier) en Bara et Antaisaka

Hyparrhenia cymbaria (Stapf).. - Verovato (vero de pierre) en Betsileo ; Verombato en Bara.

Hyparrhenia lecomtei (Stapf). - Vero en Betsileo. Hyparrhenia rufa (Stapf). - Vero en Betsileo; Vereboboka en Bara et Safisoro

Hyparrhenia ruprechtii (Fourn.). - Vero en Betsileo. Imperata cylindrica (L.). - Tenina, seul nom vernaculaire pour l'ensemble de la province.

Ipomea pes caprae (convolvulacée). - Lalandana ou Vahindalandana en Betsimisaraka, Antambahoaka.

Leersia hexandra (Sw.). - Vilona (herbe) en Betsileo, Tanala et Betsimisaraka; Tsingirifiry en Bara et Antaisaka.

Melinis minutiflora (P. de B.). - Andrasily, Sandrahirika en Bara; Menakapaha (graisse de chat sauvage) en Betsileo.

Opuntia ficus indica var. inermis. - Raiketa.

Oxyrhachis gracillima (Hubb.). - Ahitsorohitra en Betsileo.

Panicum brevifolium (Trin.). - Ahipody (herbe du Cardinal) en Tanala, Pntaisaka. Antaimoro, Betsimisaraka.

Panicum maximum (Jac.i.). - Fataka, pour loutes les populations de la province

Panicum umbellatum (Trin.). - Vnlonondry, Volonaondry (poils de mouton) en Antaisaka, Betsimisaraka, Antambahoaka.

Paspalum conjugatum (Berg). - Ahipisaka (herbe plate).

Passiflora fotida (Linne) (Passifloracée). - Tsipcapoaka en Antambahoaka.

Pennisetum pseudotriticoïdes (A. Camus), P. triticoïdes (Baker). - Horompotsy en Betsileo.

Pennisetum polystachyum (Schum.). - Rambonalika (queue de chien) en Hova; Veroboboka en Tanala et $\Lambda$ ntaisaka; Veromena (vero rouge) en Bara.

Phragmites mauritianus (Kunth.). -- Bararata, en Btesileo et Bara; Volotara en Tanala.

Rottboellia exaltata (L.). - Tsanganday en Hova, Betsileo et Bara.

Schizachyrium ambalavense ( $\AA$. Camus). - Ahitsorohitra en Betsileo.

Schizachyrium brevilolium (Nees). - Ahitsorohitra en Betsileo.

Schizachyrium semiherbe (Nees). - Ahitsorohitra 
(herbe à: alouette) en Betsileo; Ahiborisoa (herbe courte et bonne) en Bara.

Setaria pallidefusca (Stapf et Hubb)). - Taindalitra en Hova.

Sida rhombifolia (Malvacée). - Sandahory en Tanala; Samborimalama (difficile à saisir) en Antaimoro; Kididitsy; Tsindisy en Bara et Antaisaka.

Sorghum verticilliflorum (Stapf). - Bakaka, en Bara et Betsileo.

Sporobolus indicus (R. Br.). - Horona en Betsileo. Stenotaphrum dimidiatum (Br.). - Ahipisaka.

Tephrosia purpurea (Personn) (Légumineuse). Aingitra en Betsimisaraka et Antambahoaka; avec cette plante les autochtones préparent une teinture noire.

Trachypogon polymorphus (Hackel): - Horo en Bara; Danga pour certains Betsileo des districts d'Ambositra, de Fandriana et d'Ambatofinandrahana.
Trichopteryx stipoïdes (Hackel). - En pays Betsileo, simplement appelé, dans bien des cas : Bozaka (herbe); Rodrotra, Kirodrotra ou Tsirodrotra, Ahidambo ou Danga, dans certains cantons des districts d'Ambohimahasoa, d'Ambositra et d'Ambatofinandrahana; Kilailay à Fandriana ainsi que dans la région nord du district; Berambo qui signifie " grande queue », par les Bara. Sans nom vernaculaire bien défini, souvent confondu avec d'autres espèces, nous avons préféré, pour l'établissement de notre carte, nous abstenir de toute dénomination locale pour le désigner.

Usena lobata (L.) (Malvacée). - Tsipaka en Bara, Antaisaka et Antaimoro; Tsingaka, Tsipiaka en Tanala.

Vigna angivensis (Baker) (Légumineuse). Kimaotra ou Kimaotsa en Hova et Betsileo.

Zoissia tenuifolia (Wild.). - Volonondry en Antaifasy.

TABLEAU II. - CLIMATOLOGIE DE RÉGIONS TYPES A DANGA ET VERO (1)

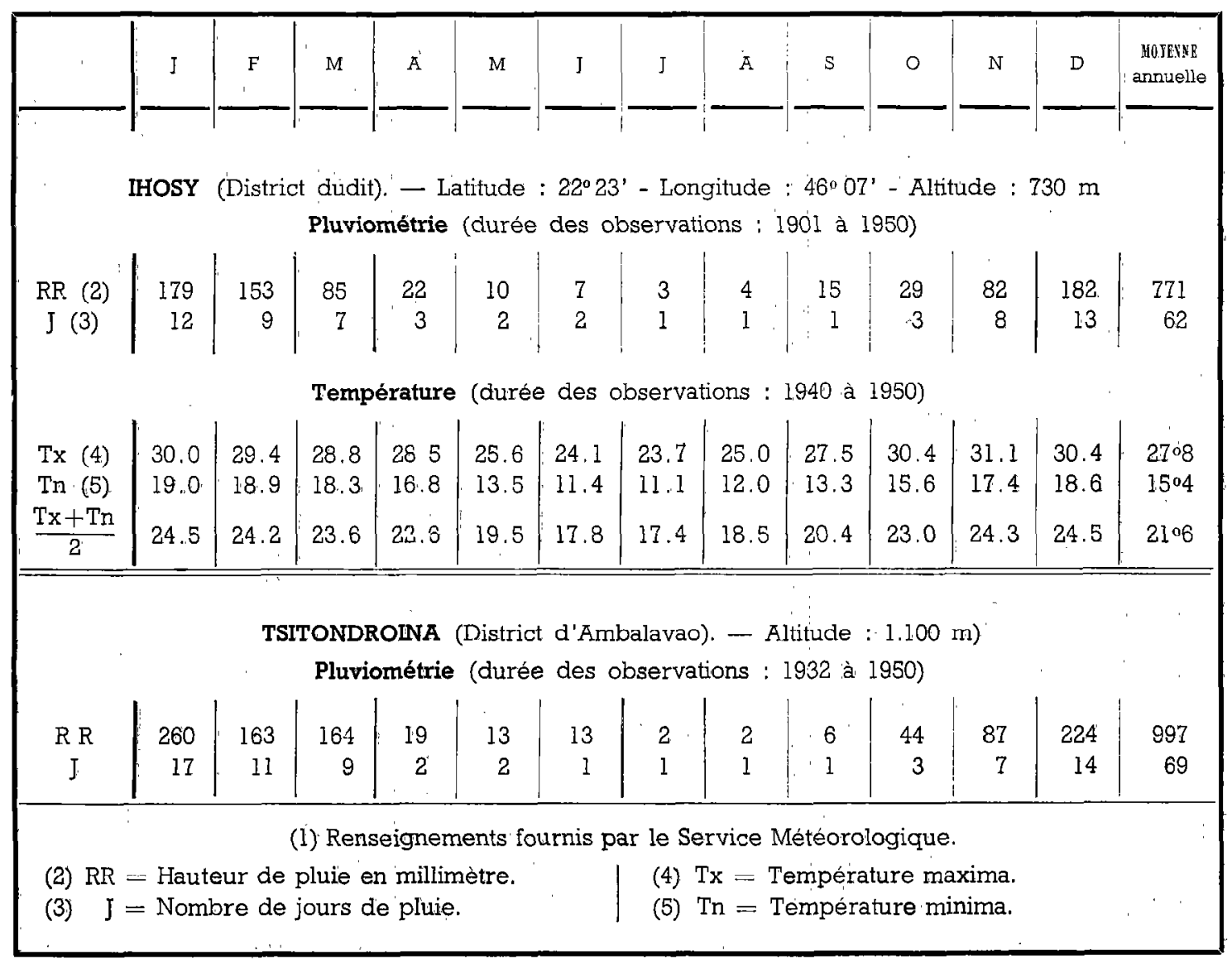




\section{TAALEEO III. - COMPOSITION DE PLANTES RÉCOLTÉES APRÈS FRUCTIFICATION DANS DES PATURAGES A DANGa ET VERO}

\begin{tabular}{|c|c|c|c|c|c|c|}
\hline & $\begin{array}{r}\text { DANGA- } \\
\text { (Heteropog }\end{array}$ & $\begin{array}{l}\text { IDAMBO } \\
\text { contortus) }\end{array}$ & $\begin{array}{r}\mathrm{v} \\
\text { (Hyparr }\end{array}$ & $\begin{array}{l}0 \\
\text { uia rufal }\end{array}$ & $\begin{aligned} & \mathrm{H} \\
&(T r a c t \\
& \text { polym }\end{aligned}$ & $\begin{array}{l}\text { O } \\
\text { ogon } \\
\text { phus) }\end{array}$ \\
\hline & Frais & Sec & Frais & Sec & Frais & Sec \\
\hline Humidité pour $100 \mathrm{~g} . .$. & 10,70 & 0 & 10,30 & 0 & 9,62 & 0 \\
\hline Cendres $\ldots \ldots \ldots \ldots \ldots \ldots \ldots \ldots$ & 8,31 & 9,31 & 8,30 & 9,23 & 3,42 & 3,78 \\
\hline Matières azotées.................. & 2,68 & 3,00 & 2,18 & 2,44 & 2,75 & 3,04 \\
\hline Matières grasses $\ldots \ldots \ldots \ldots \ldots \ldots$ & 0,15 & 0,17 & 0,14 & 0,15 & 0,26 & 0,28 \\
\hline Matières hydrocarbonées . . . . . . . . & 37,18 & 41,63 & 37.48 & 41.98 & 42,69 & 47,27 \\
\hline Débris cellulosiques $\ldots \ldots \ldots \ldots \ldots$ & 40,98 & 45,89 & 41,20 & 46,14 & 41,26 & 45,63 \\
\hline 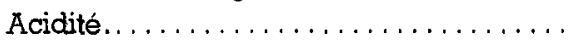 & 0,15 & 0,17 & 0,16 & 0,18 & 0.17 & 0,19 \\
\hline $\mathrm{P}^{2} \mathrm{O}^{s} \ldots \ldots \ldots \ldots \ldots \ldots \ldots \ldots \ldots \ldots \ldots$ & 0,025 & 0,028 & 0,055 & 0,062 & 0,032 & 0,035 \\
\hline $\mathrm{K}^{2} \mathrm{O} \ldots \ldots \ldots \ldots \ldots \ldots \ldots \ldots \ldots \ldots \ldots \ldots \ldots$ & 0.648 & 0,726 & 0,496 & 0,648 & 0,283 & 0,315 \\
\hline $\mathrm{CaO} \ldots \ldots \ldots \ldots \ldots \ldots \ldots \ldots \ldots \ldots \ldots$ & 0,030 & 0,033 & 0,028 & 0,031 & 0,014 & 0,015 \\
\hline Valeur énergétique en calories pour & & & & & & \\
\hline $100 \mathrm{~g} \ldots \ldots \ldots \ldots \ldots \ldots \ldots \ldots \ldots \ldots$ & 159,03 & $178,1 !$ & 158,28 & 177,27 & 182,13 & 200,34 \\
\hline
\end{tabular}

TABLEAU IV. - CLIMATOLOGIE DE RÉGIONS TYPES A KIFAFA ET TRICHOPTERYX

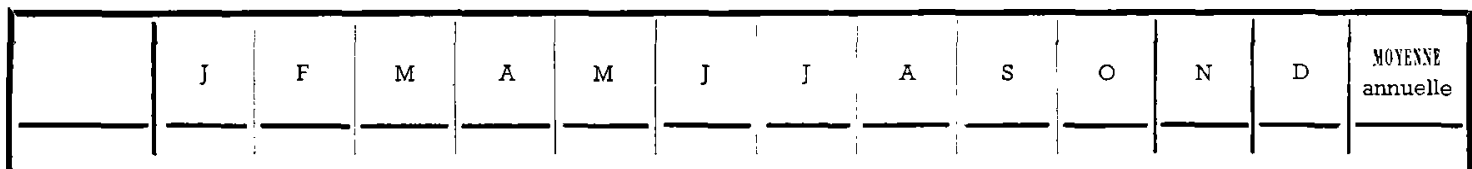

AMBOSITRA (District dudit). - Latitude : $20^{\circ} 32^{\prime}$ - Longitude : $47^{\circ} 14^{\prime}$ - Altitude : $1.345 \mathrm{~m}$

Pluviométrie (durée des observations : 1902 à 1950)

\begin{tabular}{c|r|r:r|r|r|r|r|r|r|r|r|r|r}
$\mathrm{RR}$ & 290 & 257 & 215 & 83 & 38 & 19 & 27 & 20 & 16 & 64 & 167 & 276 & 1.472 \\
$\mathrm{~J}$ & 22 & 19 & 20 & 13 & 10 & 9 & 12 & 8 & 6 & 8 & 15 & 21 & 163
\end{tabular}

Température (durée des observations : 1940 à 1950)

\begin{tabular}{c|r|rr|r|r|r|r|r|r|r|r|r|r|}
$\operatorname{Tx}$ & 25.8 & 25.6 & 25.0 & 24.2 & 22.0 & 20.4 & 19.2 & 20.1 & 22.3 & 25.2 & 26.2 & 26.0 & 2305 \\
$\operatorname{Tn}$ & 15.9 & 15.6 & 15.3 & 13.8 & 11.3 & 9.3 & 8.5 & 8.5 & 9.5 & 11.7 & 13.8 & 15.1 & 1203 \\
$\operatorname{Tx}+\operatorname{Tn}$ & 20.8 & 20.6 & 20.1 & 19.0 & 16.7 & 14.8 & 13.9 & 14.3 & 15.9 & 18.4 & 20.0 & 20.5 & 1709 \\
\hline 2 & 20.8 & & & & & &
\end{tabular}

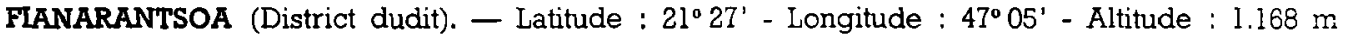
Pluviométrie (durée des observations : 1902 à 1950)

\begin{tabular}{c|r|rrr|r|r|r|r|r|r|r|r|r|r}
$\mathrm{R} \mathrm{R}$ & 273 & 243 & 143 & 51 & 38 & 17 & 21 & 18 & 22 & 49 & 128 & 246 & 1.249 \\
$\mathrm{~J}$ & 18 & 18 & 16 & 11 & 9 & 6 & 9 & 7 & 6 & 7 & 12 & 17 & 136
\end{tabular}

Température (durée des observations : 1940 à 1950)

\begin{tabular}{c|c|c|c|c|c|c|rrr|r|r|r|c|}
$\operatorname{Tx}$ & 26.1 & 25.6 & 25.3 & 24.3 & 21.8 & 20.3 & 18.8 & 20.7 & 22.4 & 25.5 & 27.0 & 26.5 & 2306 \\
$\operatorname{Tn}$ & 15.5 & 16.2 & 15.9 & 14.6 & 11.9 & 10.1 & 9.6 & 9.7 & 10.6 & 12.7 & 14.7 & 15.7 & 1301 \\
\hline $\operatorname{Tx}+\operatorname{Tn}$ & 21.3 & 20.9 & 20.6 & 19.4 & 16.9 & 15.2 & 14.2 & 15.2 & 16.5 & 19.1 & 20.8 & 21.1 & 1804 \\
\hline
\end{tabular}




\section{TABLEAU V. - COMPOSITION DE PLANTES RÉCOLTÉES APRÈS FRUCTIFICATION} DANS DES PATURAGES A KIFAFA ET TRICHOPTERYX

\begin{tabular}{|c|c|c|c|c|}
\hline & (Arist & caulis). & $\begin{array}{r}\text { HC } \\
\text { SPennisetut }\end{array}$ & $\begin{array}{l}\text { SY } \\
\text { otriticoídes) }\end{array}$ \\
\hline & Frais & Sec & Frais & Sec \\
\hline Humidité pour $100 \mathrm{~g}$. & 10,76 & 0 & 10,16 & 0 \\
\hline Matières azotées ..... & 2,5 & 2,8 & 2 & 2,22 \\
\hline Matières grasses.............. & 1,14 & 1,27 & 0,78 & 0,865 \\
\hline Matières hydrocarbonées........... & 43,72 & 49,08 & 44,25 & 49,41 \\
\hline Débris cellulósiques............. & 36,6 & 40,95 & 37,58 & 41,72 \\
\hline Acidité $\ldots \ldots \ldots \ldots \ldots \ldots \ldots \ldots \ldots \ldots$ & 0,104 & $»$ & 0,101 & $»$ \\
\hline Matières minérales.................... & 5,28 & 5,90 & 5,22 & 5,79 \\
\hline $\mathrm{P}^{2} \mathrm{O}^{5}$ en grammes pour $100 \mathrm{~g} . \ldots \ldots \ldots$ & 0,478 & 0,535 & 0,189 & 0,21 \\
\hline 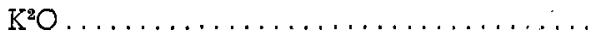 & 1,916 & 2,14 & 0,837 & $0,92,9$ \\
\hline 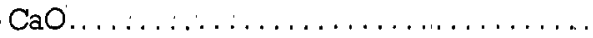 & 0,293 & 0,328 & 0,29 & 0,322 \\
\hline
\end{tabular}

\section{TABLEAU VI - CLIMATOLOGIE DE RÉgIONS TYPES a DANGA, VERO, IIFAFA ET TRICHOPTERYX}

\begin{tabular}{|c|c|c|c|c|c|c|c|c|c|c|c|c|c|}
\hline & J & $\mathrm{F}$ & M & A & M & J & J & A & $\mathrm{s}$ & 0 & $\mathrm{~N}$ & D & $\begin{array}{c}\text { MloveNXP } \\
\text { annuelle }\end{array}$ \\
\hline \multicolumn{14}{|c|}{$\begin{array}{l}\text { IVOHIBE (District de Farafangana). - Altitude : } 930 \mathrm{~m} \\
\text { Pluviométrie (durée des observations : } 1935 \text { à 1950) }\end{array}$} \\
\hline R R & 179 & 230 & 149 & 21 & 26 & 24 & 28 & 17 & 12 & 30 & 73 & 152 & 941 \\
\hline $\mathrm{J}$ & 19 & 17 & 16 & 8 & 8 & 8 & 10 & 7 & 6 & 6 & 12 & 17 & 134 \\
\hline \multicolumn{14}{|c|}{$\begin{array}{l}\text { IAKORA (District d'IHOSY) - Altitude : } 730 \mathrm{~m} \\
\text { Pluviométrie'(durée des observations : } 1940 \text { a 1950) }\end{array}$} \\
\hline R R & 261 & 237 & 139 & 44 & 20 & 25 & 15 & 13 & 7 & 42 & 130 & 242 & 1175 \\
\hline$J$ & 16 & 14 & 14 & 7 & 5 & 0 & 5 & 4 & 3 & 6 & 13 & 15 & 108 \\
\hline
\end{tabular}


TABLEAd VII. - CLIMATOLOGIE DE RÉgIONS TYPES a AHIPISAKa ET TENINA

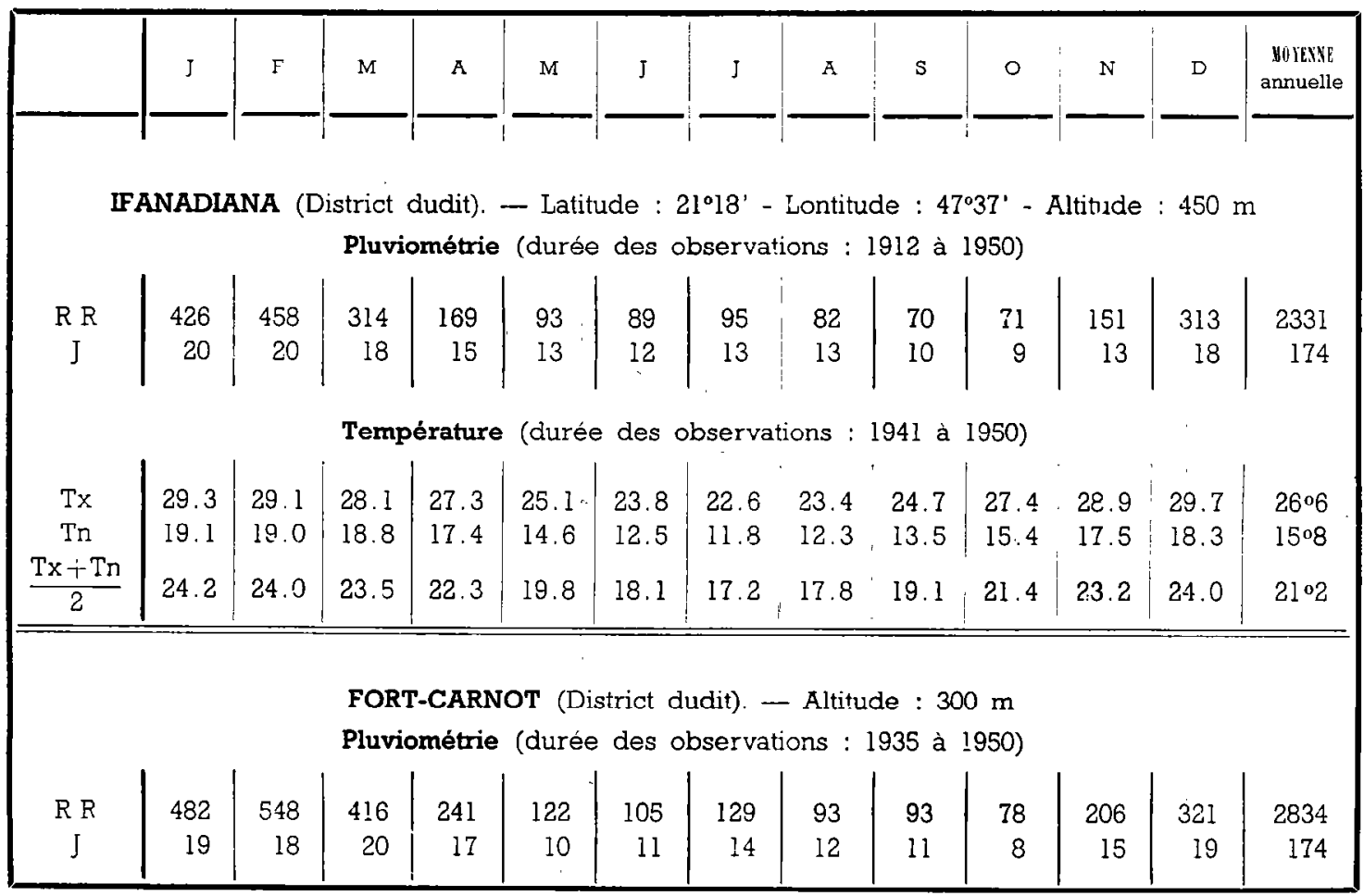


TABLEAU VIII. — CLIMATOLOGIE DE RÉGIONS TYPES A AHIPISAKA, TENINA, KIFAFA ET VERO

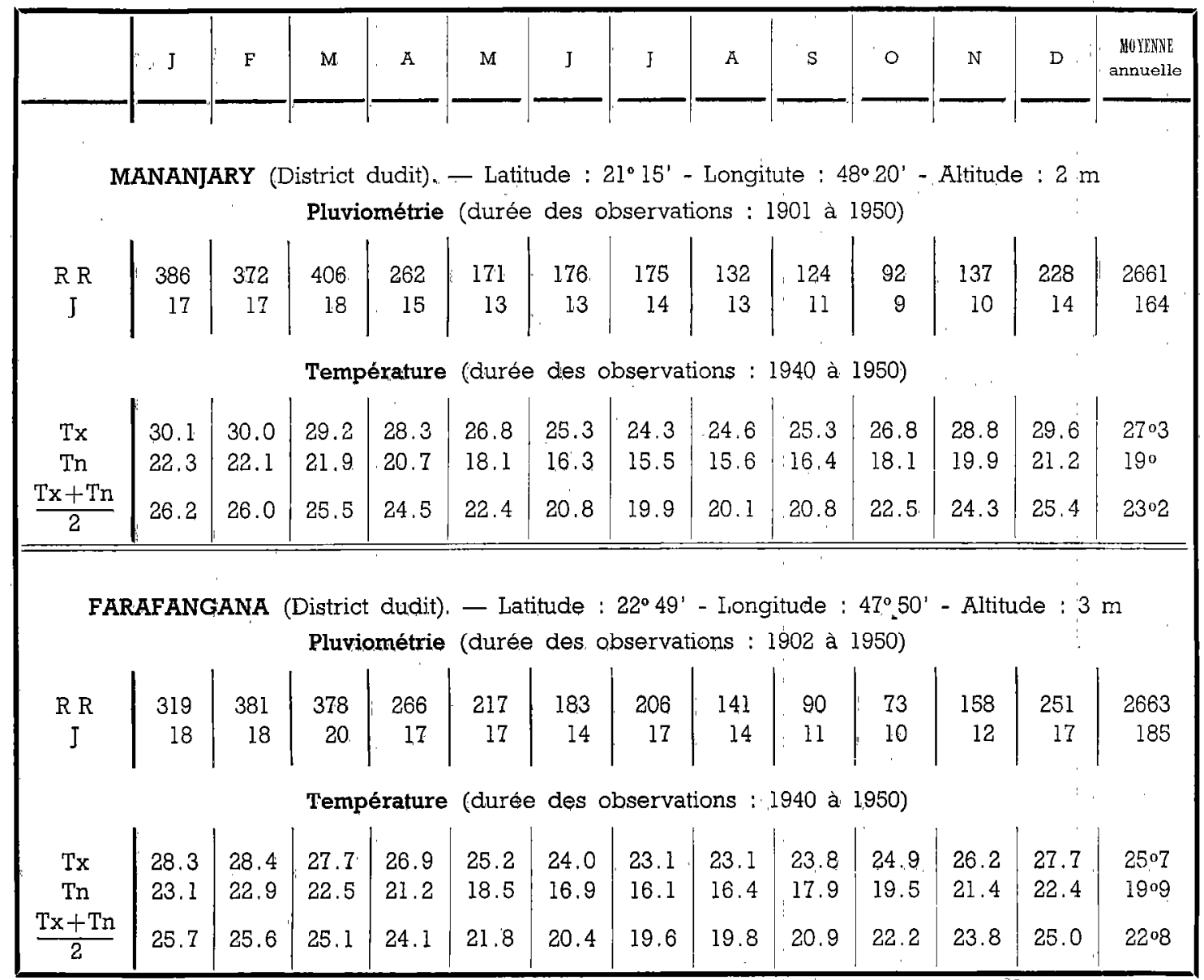

